\title{
Artritis Reumatoide Juvenil. Análisis clínico de siete casos
}

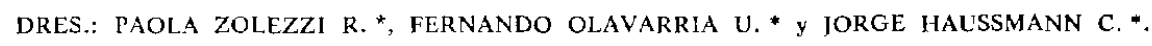

En el grupo de las enfermedades del mesénquima en el niño, la Artritis Reumatoide Juvenil (ARI) ocupa un lugar preponderante (1).

Es de todos conocido lo insidioso y proteiforme de sus manifestaciones clínicas y la falta de elementos de laboratorio que permitan asegurar con certeza el diagnóstico de ARJ, lo que crea problemas de diferenciación con las otras enfermedades del mesénquima y también con alguna frecuencia con otros cuadros clínicos de muy diferente etiopatogenia $(2,3,4)$.

Nos ha parecido de interés presentar siete casos de ARJ reunidos en los últimos dos años en el Servicio de Pediatría, Hospital Regional, Valdivia.

Material. y METodo. Entre Julio de 1972 y Junio de 1974, egresaron de la Unidad de Medicina Infantil del Servicio de Pediatría del Hospital Regional de Valdivia siete niños con diagnóstico de Artritis Reumatoide Juvenil $(0,9 \%$ del total de egresos).

El diagnóstico de ARJ se basó en el análisis global del cuadro clínico de los exámenes de laboratorio, de las alteraciones radiológicas y de las alteraciones histopatológicas.

Cuadro clínico: edad, sexo, forma de comienzo, diversas manifestaciones de compromiso articular, rigidez matinal, aparición de exantema, compromiso del estado general, fiebre, adenopatías, existencia de nódulos subcutáneos, esplenomegalia, compromiso ocular y alteración del crecimiento $(1,2,3,5)$.

Exámenes de laboratorio: en que se valoró la existencia de anemia, leucocitosis, sedimentación elevada, hiperglobulinemia con inversión del índice Alb/Glob., positividad o negatividad del factor reumatoide, negatividad del estudio seriado de células de lupus (6).

\footnotetext{
* Instituto de Pediatría. Universidad Austral.

Servicio de Pedlatría. Hospital Regional de Valdivia.

Trabajo presentado a las Cuartas Jornadas Anuales de Pediatría, Valdivia, Octubre de 1974.
}

Alteraciones radiológicas: consistentes fundamentalmente en uno o más de los siguientes signos: aumento de volumen de partes blandas, articulares o periarticulares, osteoporosis, disminución del espacio interóseo, subluxaciones y desviaciones del eje y erosiones óseas $(7,8,9)$.

Alteraciones histopatológicas: encontradas en la biopsia articular en que destaca la hipertrofia y engrosamiento de la sinovial, los infiltrados densos de linfocitos y células plasmáticas, propagación de fenómenos inflamatorios sinoviales a tejidos adyacentes con posterior formación del pannus y su proliferación con destrucción del cartílago articular. En la biopsia de nódulo subcutáneo se investigó el aspecto característico del granuloma reumatoídeo $(10)$.

RESUlTAdos y Discusion. Edad y sexo: La edad de comienzo de la ARJ en nuestros 7 casos

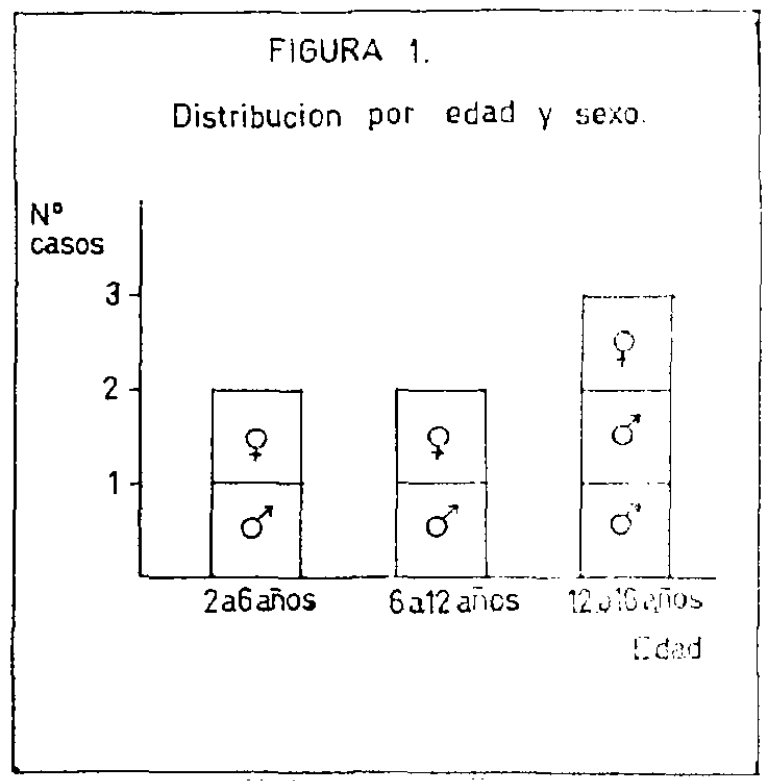


varía entre los 5 y los 14 años de edad, con un promedio de 9,6 años. En la figura 1 se observa la distribución por edad y sexo.

Se describe la ARJ a cualquier edad siendo más frecuente entre los 2 y 4 años, volviendo a presentar una mayor incidencia en la pubertad (2, 3. 5, 11). En nuestros casos llama la atención el no observar ningún paciente menor de 5 años.

Se ha descrito que la incidencia de la ARJ es mayor en el sexo femenino, especialmente en el grupo de adolescentes (5), hecho que no hemos observado, probablemente debido al escaso número de pacientes.

Forma de comicnzo: en Cinco de nuestros pacientes se manifestó por poliartritis, asociada a diferentes manifestaciones sistémicas, tales como fiebre elevada, rash, palidez, nódulos subcutáncos, poliadenopatías, pericarditis y compromiso del estado general moderado o severo. En cuatro de ellos el comienzo fue agudo y en uno de tipo insidioso (Figura 2).

El comienzo poliarticular más el compromiso sistémico ya sea de comienzo agudo o insidioso se describe en otras series en el $40 \%$ de los casos (2) .

Los dos pacientes que inician su cuadro en forma insidiosa corresponden al grupo de adolescentes lo que se describe en el $56 \%$ de los pacientes cuya sintomatología comienza después de los 12 años asemejándose a la artritis reumatoide del adulto (5).

En dos pacientes la forma de comienzo es monoarticular, agregándose artritis de grandes articulaciones un mes después sólo en uno de cllos.

En el 30\% de los pacientes con ARI el episodio inicial es monoarticular agregándose a las pocas semanas o meses el compromiso de las otras

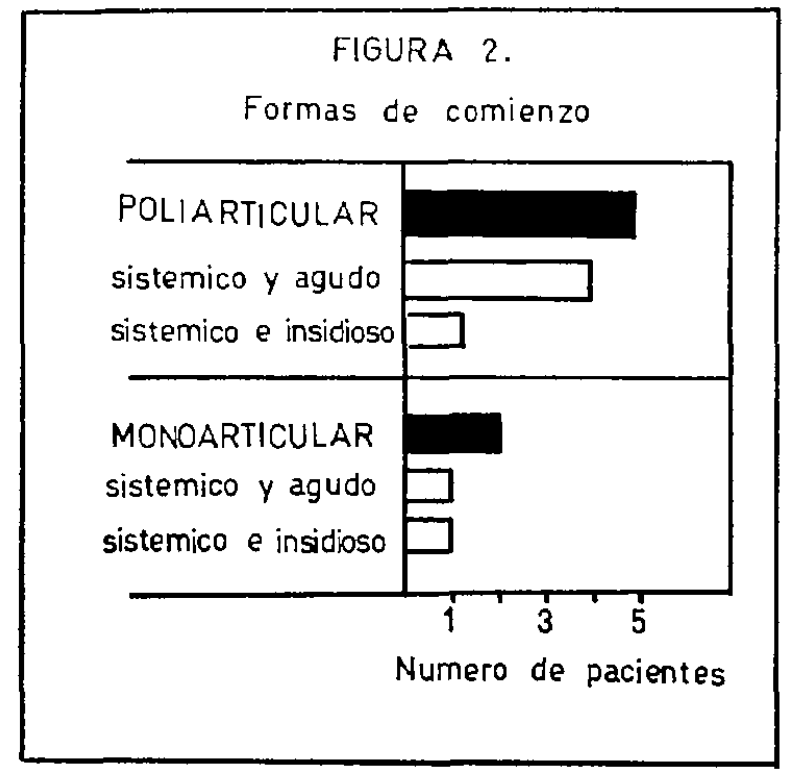

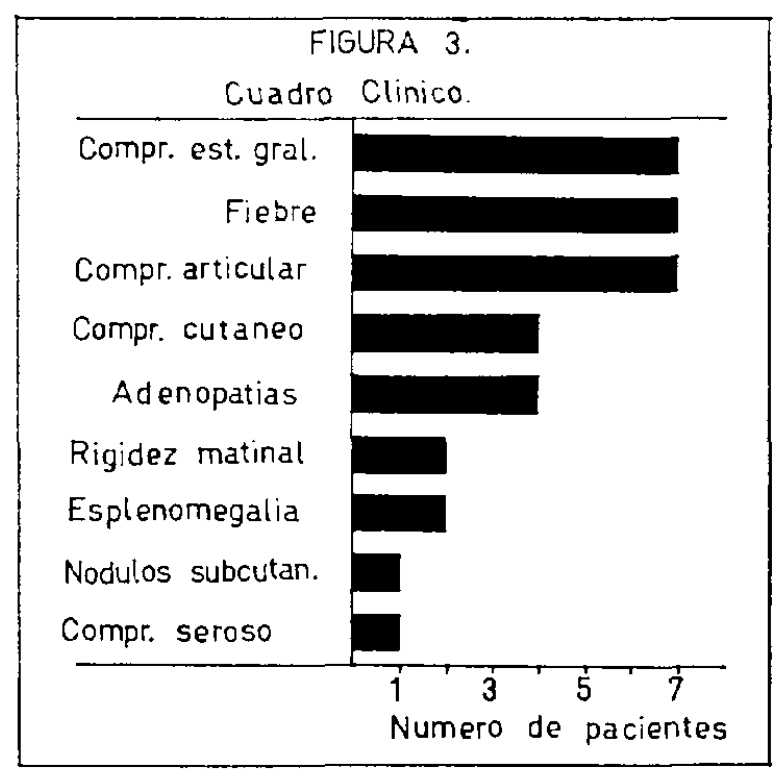

articulaciones, pero en el $10 \%$ de estos pacientes el compromiso continúa siendo monoarticular, generalmente de grandes articulaciones: rodillas, coxo-femorales, codos (2).

Cuadro clinico: En la figura 3 se muestran las manifestaciones más importantes, destacando por su frecuencia el compromiso del estado general, la fiebre, el compromiso articular, el compromiso cutáneo y las adenopatías; con menor frecuencia se observó rigidez matinal, esplenomegalia, nódulos subcutáneos y compromiso de serosas.

El compromiso del estado general fue severo en cinco pacientes acompañándose de astenia, apatía y anorexia con baja de peso.

La fiebre fue un hallazgo constante en los siete pacientes, adquirjendo en cinco de ellos carácter intermitente. La incidencia de fiebre en ARJ varía entre 62 a $90 \%(1,2,12)$.

Compromiso articular: Se manifestó en todos los pacientes por artralgias y artritis con compromiso de grandes y pequeñas articulaciones. como se observa en la figura 4 , sicndo las más comúnmente afectadas las articulaciones radiocarpianas, rodillas, hombros y tobillos.

En seis pacientes la duración del compromiso articular fue superior a los tres meses, manifestándose por artritis de cuatro o más articulaciones.

Dentro de las manifestaciones articulares, merece destacarse la intensidad de las artralgias, el aumento del volumen articular, especialmente periarticular, con deformación del contorno articular y la impotencia funcional de grado variable que en cinco casos impidió la deambulación en algún momento de su evolución.

Se observó atrofia de la musculatura de la mano en tres casos y atrofia de la musculatura de las extremidades inferiores en otros dos casos. 
FIGURA 4

Incidencia de compromiso articular.

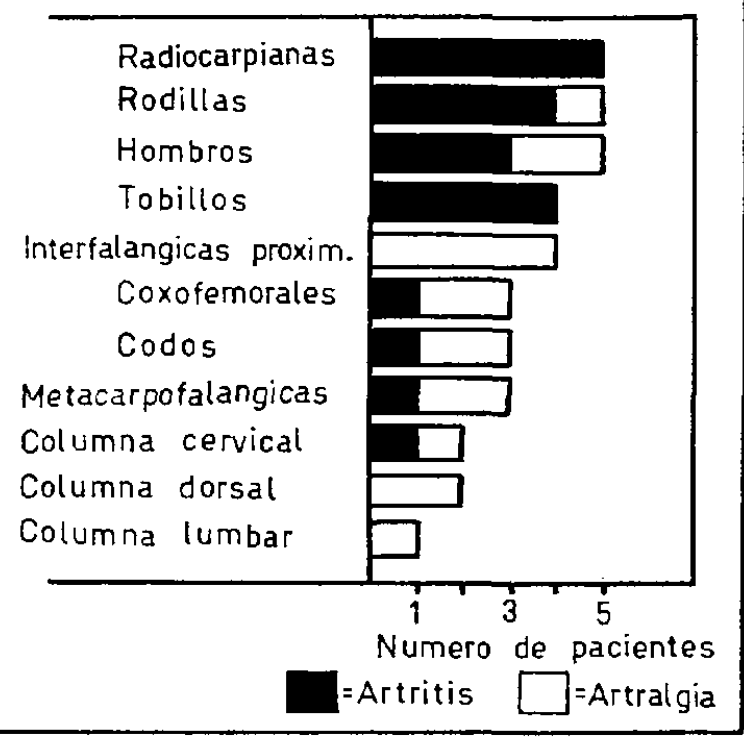

Una notoria rigidez matinal se observó en dos pacientes. Estas observaciones coinciden con las clásicas descripciones de Ansell y Bywaters (3), Calabro y Marchesano (1) y Brewer (2).

Compromiso cutáneo: En cuatro pacientes observamos erupción máculo-papulosa eritematosa, de distribución predominante en tronco y en extremidades superiores; en dos pacientes comprometió la cara, en dos casos fue recidivante presentándose en dos y tres brotes fugaces durante el período febril.

Se ha descrito compromiso cutáneo de variadas formas en ARJ entre un 20 y un $70 \%$, predominando el eritema de tipo máculopapuloso (13, 14, 15).

Compromiso ganglionar: Cuatro pacientes presentaron poliadenopatías generalizadas predominando el compromiso cervical, axilar e inguinal. Revisando diferentes series el promedio de compromiso ganglionar en ARJ es de un $23 \%(2,3$, $14,15)$, siendo su duración variable, días o meses. El aumento o la disminución de los ganglios iría paralelo a la actividad de la enfermedad (16).

Esplenomegalia: Pesquisamos esplenomegalia moderada en dos pacientes, presentándose este hecho entre un 10 y un $33 \%$ en otras series $(2,3)$.

Nódulos subcutáneos: Uno solo de nuestros pacientes de sexo masculino, de 14 años, presentó numerosos nódulos subcutáneos y yuxtaarticulares, indoloros, no adheridos, en hombros, codos, muñecas, interfalángicas proximales y en las apófisis espinosas. Este hecho se describe en un 2 a un $12 \%$ de casos de ARJ, siendo más frecuente en el adulto $(2,3,14,15,17)$.
Compromiso de serosas: Un paciente presentó poliserositis con pericarditis, derrame pleural bilateral y poliartritis. Se descartó un lupus eritematoso diseminado y una enfermedad reumática activa. La asociación de pericarditis con derrame pleural parece ser la manifestación más frecuente del compromiso pleuropulmonar en ARJ, habitualmente pequeño o moderado y en muchos casos es asintomático.

La pericarditis se describe en un $7 \%$ a $10 \%$ de los casos de $\operatorname{ARJ}(1,2,3)$, pudiendo ser de tipo leve, moderado o severo. Sin embargo, los exámenes post-mortem revelan una incidencia del $45 \%$ (19) .

\section{Laboratorio:}

Sedimentación: Todos los pacientes presentaron sedimentación elevada, en cinco casos sobre $100 \mathrm{~mm}$. en una hora. En el examen que con mayor frecuencia se altera en ARJ, representando un excelente índice de actividad de la enfermedad, aun cuando puede ser normal en pacientes mayores o monoarticulares (6).

Hemograma: En la serie roja se observó anemia hipocroma microcítica de moderada intensidad, en tres pacientes. Lo común es encontrar anemia normocítica de moderada intensidad, lo que se observa en el $40 \%$ de los casos de ARJ (2, 20,21).

En la serie blanca, seis pacientes mostraron una leucocitosis superior a 10.000 elementos por $\mathrm{mm}^{3}$, con neutrofilia y moderada desviación a izquierda. Se describe este hecho en $50 \%$ de los casos, con mayor frecuencia que en el adulto, constituyéndose en el hallazgo más importante del hemograma en ARJ $(1,6,17)$.

Sedimento urinario: Los exámenes seriados de orina revelaron en un solo paciente proteinuria leve sólo durante la fase febril (6).

Electroforesis de proteínas plasmáticas: Se practicó en cuatro pacientes encontrándose una disminución franca de la fracción albúmina, apareciendo como fenómeno constante el aumento de la fracción globulina en base a un aumento leve o moderado de alfa uno y beta, un aumento marcado de alfa dos y aún mayor de las gama globulinas con inversión del índice Alb/Glob.

La electroforesis de proteínas plasmáticas se considera de extrema utilidad para establecer la presencia de inflamación en pacientes con ARJ aunque se desconoce la verdadera causa del aumento de las globulinas. En el $30 \%$ de los pacientes se encuentra una disminución marcada de las albúminas séricas $\mathrm{y}$ en el $50 \%$ un aumento de la gama globulina superior a $1,2 \mathrm{grs} \%$. En la literatura no se describen aumentos de globulina alfa uno y en número muy escaso de niños se ha 
T A B L A 1

ELECTROFORESIS DE PROTEINAS

\begin{tabular}{lccccc}
\hline & $\begin{array}{c}\text { Albú- } \\
\text { mina }\end{array}$ & Alfa 1 & Alfa 2 & Beta & gama \\
\hline Valores & 55,9 & 4,7 & 9,9 & 13,9 & $\mathbf{1 5 , 5}$ \\
normales & $\pm 3,2$ & $\pm 1,3$ & $\pm 1,5$ & $\pm 1,5$ & $\pm 0,9$ \\
& & & & & \\
MRS & 13 & 6 & 18 & 15 & 43 \\
VSS & 18,7 & 10,7 & 17,3 & 16,7 & 36,6 \\
PFC & 24 & 11,5 & $\mathbf{1 7}$ & $\mathbf{1 5 , 5}$ & 32 \\
RNS & 22 & 6,4 & 14,8 & $\mathbf{1 4 , 8}$ & 46,8 \\
\hline
\end{tabular}

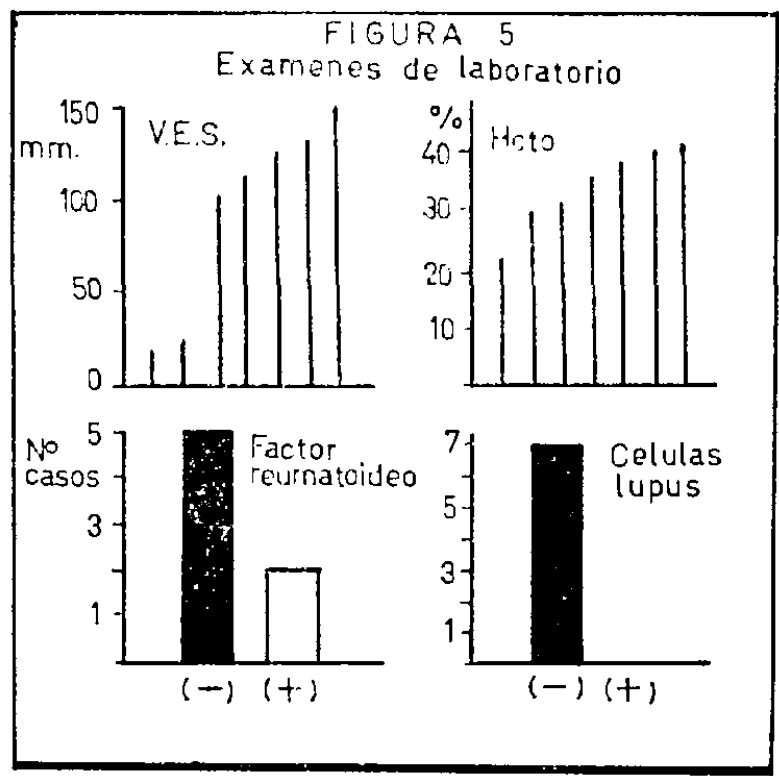

encontrado un aumento leve de globulina beta y de globulina alfa dos $(2,4)$. Tabla 1 .

Factor reumatoídeo: Practicado en todos los pacientes fue positivo sólo en dos casos (Figura 5). En alrededor del 15\% de los pacientes con ARI el factor reumatoídeo es positivo, mientras que en la artritis reumatoide del adulto su positividad alcanza al $70 \%(2,4,8)$.

Células de lupus: En los siete pacientes se hicieron estudios seriados de búsqueda de células de lupus, siendo negativa en todos ellos. La positividad de este fenómeno en ARJ ha sido descrita desde un 3 a $5 \%$ hasta un $60 \%$, lo que pone de manifiesto la mala estandarización de esta técnica $(2,4,5,20,22)$.

Electrocardiograma: Se encontraron alteraciones del trazado ECG compatibles con una pericarditis en un solo paciente.

\section{Alteraciones radiológicas:}

E1 hallazgo que observamos más frecuentemente fue el aumento de volumen de partes blandas en los sitios de compromiso articular, especialmente en las regiones interfalángicas y en rodillas, acompañándose de desmineralización yuxtaarticular en algunos casos. Con menor frecuencia observamos estrechamiento del espacio interóseo

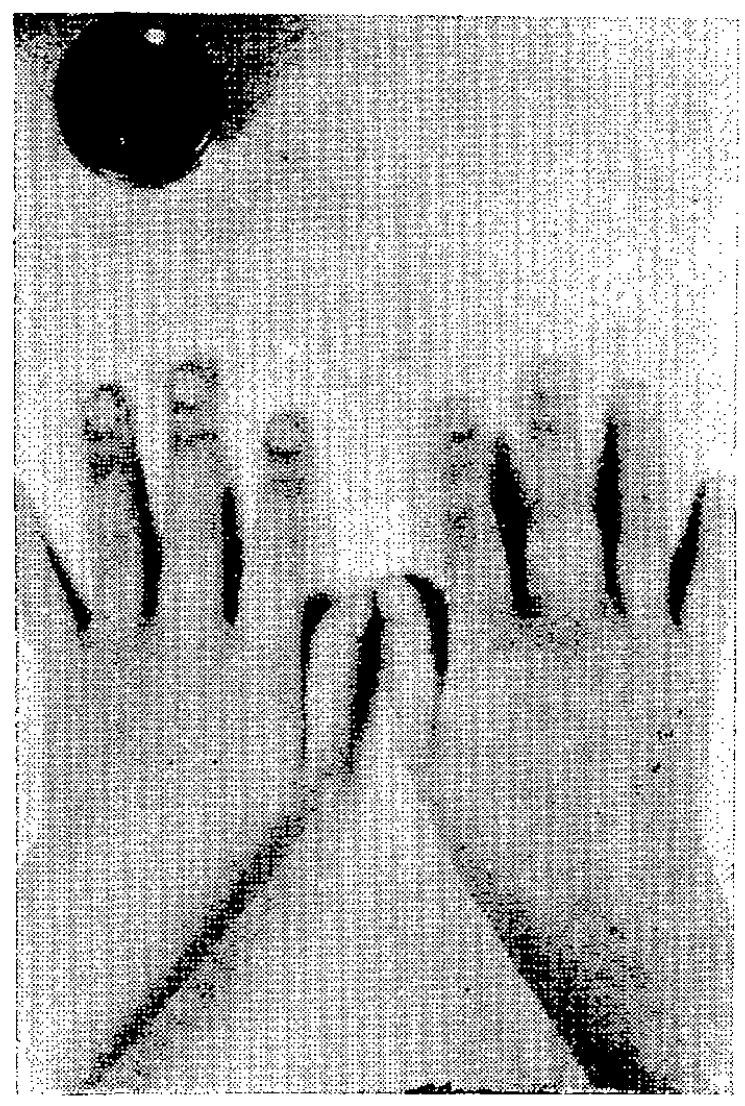

Flgurg 0.- Aumento de volumen metacarpo-falänglco.

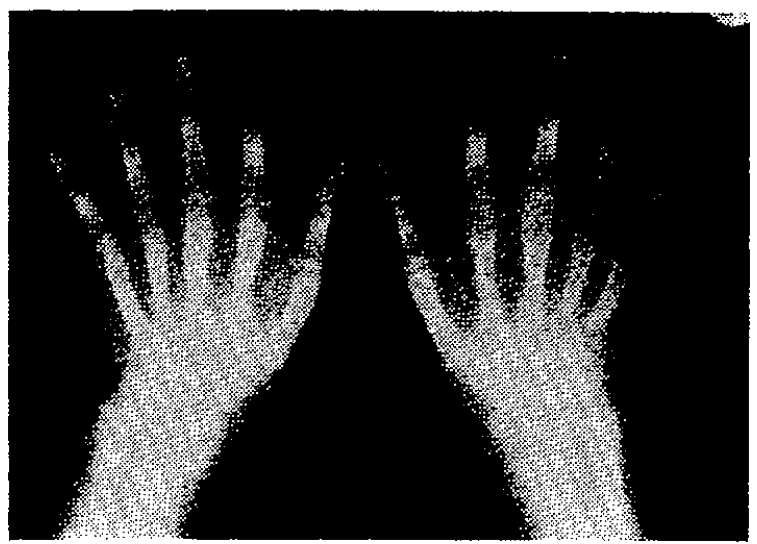

Figura 7.- Radiografía de manos correspondiente a flgura 6. 


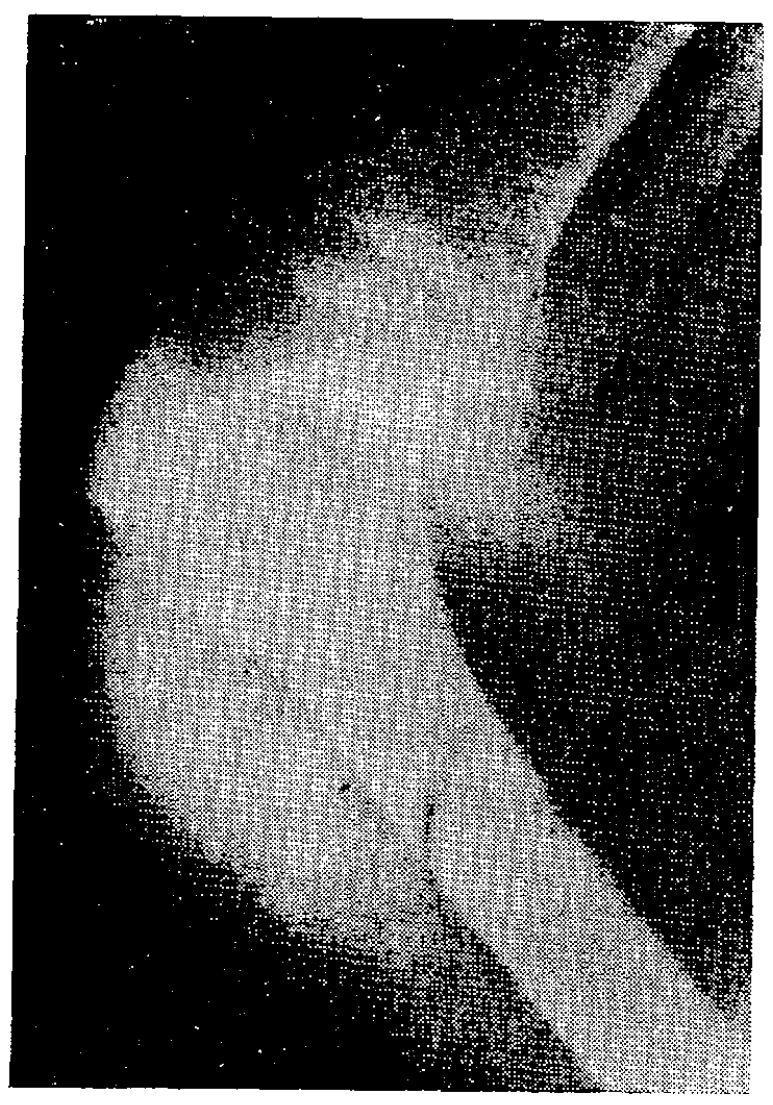

Figura 8.- Alumento de volumen de partes blandas.

especialmente en los huesos del carpo. (Figuras 6,7 y 8 ).

No hemos encontrado cambios destructivos articulares que se ven en grupos de mayor edad ni aún en los huesos del carpo donde las erosiones aparecen precozmente durante la primera época de la vida. Tampoco hemos observado las otras manifestaciones radiológicas descritas en ARJ (7, $8,9)$.

\section{Alteraciones histopatológicas:}

Biopsia articular: El estudio histopatológico de sinovial reveló densos infiltrados linfoplasmocitarios de tendencia nodular, subepitelial y perivascular y foco de necrosis fibrinoide. Las alteraciones que se observan en las Figuras 9 y 10 corresponden a las descritas para la sinovitis reumatoide.

Biopsia de nódulo subcutáneo: El estudio histopatológico de nódulo subcutáneo reveló la constitución de nódulos granulomatosos constituidos por zonas de necrosis fibrinoide alrededor de las cuales hay proliferación vascular e infiltrado linfoplasmocitario. Figuras 11 y 12.
En niños, los nódulos subcutáneos se observan en ARJ, en enfermedad reumática y en casos sin patología definida. Su histología en la ARJ difiere tanto del nódulo subcutáneo de la artritis reumatoide del adulto como del nódulo de la enfermedad reumática, aun cuando puede asemejarse más a estos últimos (23).

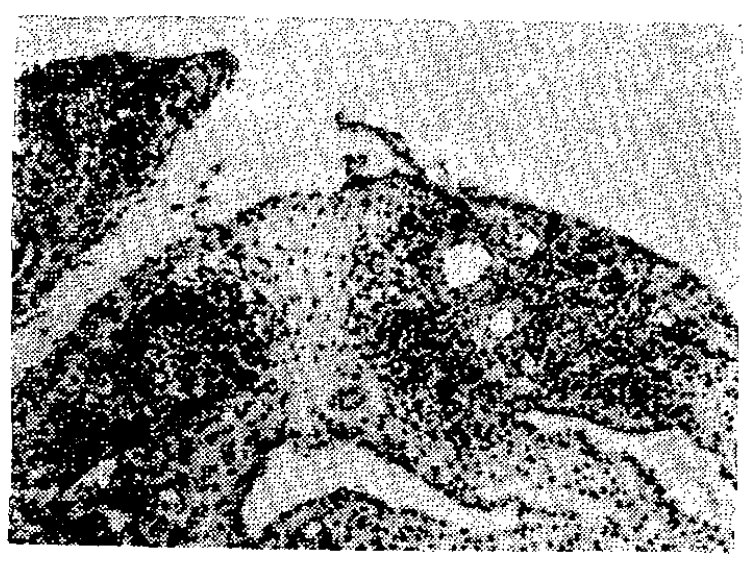

Figura 9.- Biopsia sinovlal: Infiytrados linfoplasmocitarios y focos de necrosis fibrinoide

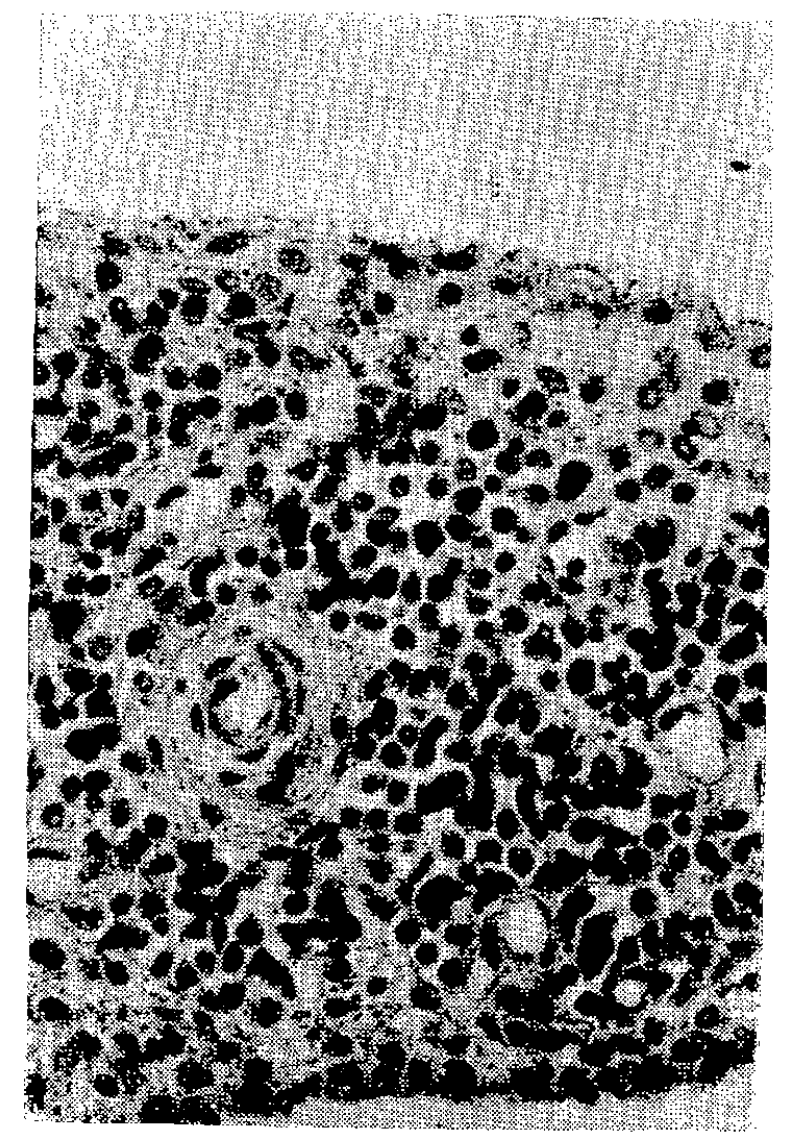

Figura 10.- Mayor aumento de 1a figura anterior. 


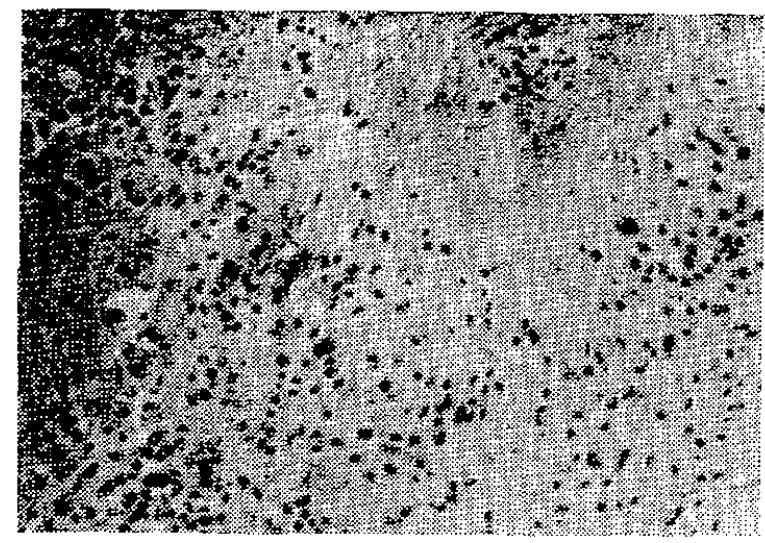

Figura 11.- Blopsia de nódulo subcutáneo: Necrosis fibrinoide y prolifcración celular.

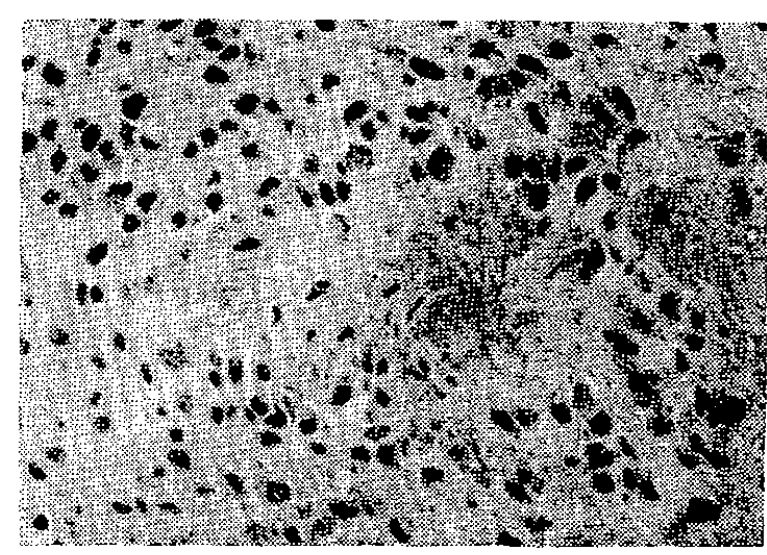

Figura 12.- Giopsia de nódulo subcutáneo: Necrosis fibrinoide.

\section{Diagnóstico diferencial:}

Los diagnósticos más comúnmente planteados correspondieron a enfermedad reumática activa, lupus eritematoso diseminado, sindrome febril prolongado y artritis séptica.

La Asociación Americana de Reumatología acepta como definitivo el diagnóstico de artritis reumatoide cuando existen cinco de los elementos que a continuación se enumeran, con una duración total mínima de seis semanas de los síntomas y signos articulares: a) rigidez matinal, b) dolor a la movilización articular c) aumento de volumen al menos de una articulación, d) aumento de volumen de más de una articulación, e) compromiso articular simétrico, f) nódulos subcutáneos, g) alteraciones radiológicas características de artritis reumatoide, h) positividad de las reacciones de aglutinación, i) precipitado débil de la mucina del líquido sinovial, j) alteraciones histopatológicas características en la biopsia sinovial y $\mathrm{k}$ ) alteraciones histológicas características en los nódulos. Kornreich, Koster y Hanson (5) modifican el criterio para ARJ incluyendo el rash típico de la ARJ y alzas térmicas diarias desde lo normal hasta 39? C o más sin evidencia de infección.

Ansell y Bywaters (3) aceptan como definitivo el diagnóstico de ARJ cuando existe una poliartritis que afecte más de cuatro articulaciones por un mínimo de tres meses y en el caso de que sean menos de cuatro articulaciones las comprometidas cuando en la biopsia de membrana sinovial existan cambios compatibles con artritis reumatoide.

De acuerdo a estos criterios de diagnóstico los enfermos estudiadus corresponden a 7 casos de ARJ.

\section{RESUMEN}

Se presenta el cuadro clinico, laboratorio, alteraciones radiológicas e histopatológicas encontradas en siete pacientes portadores de Artritis Reumatoide Juvenil.

La ARJ no es una enfermedad infrecuente en nuestro medio ya que corresponde al $0,9 \%$ de los egresos hospitalarios de la Unidad de Medicina Infantil de nuestro Servicio de Pediatría.

Nuestras observaciones coinciden con las descritas por otros autores.

Sólo el análisis conjunto del cuadro clinico, de los resultados de laboratorio, del estudio del líquido sinovial, las alteraciones radiológicas, las alteraciones histopatológicas articulares y de los nódulos subcutáneos y la evolución del cuadro clínico pueden certificar el diagnóstico de Artritis Reumatoide Juvenil.

\section{REFERENCIAS}

1.-Calabro, J. J. \& Marchesano, J. M.: "The early natural history of juvenile rheumatoid arthritis. A 10-year follow-up study of 100 cases". Med. Clin. N. Amer. 52: 567, 1968.

2.-Brewer, E. J.: "Juvenile Rheumatoid Arthritis". Ed. W. B. Saunders Company, Philadelphia, 1970, pág. 2 y sig.

3.-Ansell, B. M. \& Bywaters, E. G. L.: "Rheumatoid Arthritis (Still's disease)". Ped. Clin. N. Amer. 10: $921,1963$.

4.-Boone, J. E.; Baldwin, J. \& Levine, C.: "Juvenile Rheumatoid Arthritis". Ped. Clin. N. Amer. 21: 885, 1974.

5.-Kornreich, H.; Koster, K. \& Hanson, V.: "The rheumatic diseases in adolcscence". Ped. Clin. N. Amer. 20: 911, 1973. 
6.- Rothfield, N. F.: "Diagnosis of lupus erythematosus and rheumatoid arthritis in children". Ped. Clin. N. Amer. 18: 39, 1971.

7.-Martel, W.; Holt, J. F. \& Cassidy, J. T.: "Roentgenologic manifestations of juvenile rheumatoid arthritis". Amer. J, Roentgenol. 88: 400, 1962.

8.- Losada, M.: "Reumatismos y enfermedades afines". Ed. Andrés Bello. Santiago de Chile, 1966, pág. 248.

9.-Murray, R. O.: "Radiological changes in early Iheumatoid arthritis". Proc. Royal Soc. Med. 860: 61, 1968.

10.- Cooper, N. S.: "Pathology of rheumatoid arthritis". Med. Clin. N. Amer. 52: 607, 1968.

11.- Laaksonen, A. L.: "A prognostic study of juvenile rheumatoid arthritis. Analysis of 544 cases". Acta Pediat. Scand. Suppl. 166: 1, 1966.

12.-Rawson, A. J.; Abelson, N. M. \& Hollander, J. L.: "Studies on the pathogenesis of rheumatoid joint inflammation". Ann. Int. Med. 62: 281, 1965.

13.-Isdale, I. C. \& Bywaters, E. G. L.: "The rash of rheumatoid arthritis and Still's disease". Quart, J. Med. 25: 377, 1956.

14.-Grossman, B. J.; Ozoa, N. F. \& Arya, S. C.: "Problems in juvenile rheumatoid arthritis". Med. Clin. N. Amer. 49: 33, 1965.
15.- Schlesinger, B. E.; Forsyth, C. C. \& White, R. H.: "Observations on the clinical course and treatment of 100 cases of Still's disease". Arch. Dis. Child. $36: 65,1961$.

16.-Robertson, M. D. S.; Dudley-Hart, F.; White, D. W.; Kaki, G. \& Boardman, P. L.: "Rheumatoid lymphadenopathy". Ann. Rheum. Dis, 27: 253, 1968.

17.- Kelley, V. C.: "Rheumatoid disease in childhood". Ped. Clin. N. Amer. 7: 435, 1960.

18.-Martell, W.; Abell. M. R.; Mikkelsen, \& Whitehouse, W. M.: "Pulmonary and pleural lesions in rheumatoid disease". Radiol. 90: 641, 1968.

19.-Lietman, P. S. \& Bywaters, E. G. L.: "Pericarditis in juvenile rheumatoid arthritis". Pediat. 32: 855, 1963.

20.-Taylor, J. C.: "Hematologic manifestations of systemic disease". Ped. Clin. N. Amer. 19: 1071, 1972.

21.-Burns, S. L.: "Anemia in Rheumatoid arthritis". Međ. Clin. N. Amer. 52: 527, 1968.

22.-Hanson, V.; Kornreich. H. \& Drexler, E.: "Rheumatoid factor in children with lupus erythematosus". Amer. J. Dis. Child. 112: 28, 1966.

23.-Bywaters, E. G. L.; Glynn, L. E. \& Zeldis, A.: Subcutaneous nodules of Still's disease". Ann. Rheum. Dis. 17: 278, 1958.

Nuestros agradecimientos al Servicio de Anatomfa Patológica del Hospital Jobn Kennedy, Valdivia, por su colaboración en este trabajo. 